\title{
Guidelines for the management of depression: NICE work?
}

Tony Kendrick and Robert Peveler

\begin{abstract}
Summary
The National Institute for Health and Clinical Excellence (NICE) recently updated its guidance on managing depression, adding specific guidance for depression in people with physical illness. The guidance should help improve the targeting of treatments, although implementation of the guidance on depression in physica illness is challenging in the National Health Service (NHS) context of separate primary and secondary care services.
\end{abstract}

\section{Declaration of interest}

T.K. is Chair of a NICE Guideline Development Group on case identification, assessment and care pathways for common mental disorders. R.P. contributed to the British Association of Psychopharmacology guidelines.
Tony Kendrick (pictured) is Professor of Primary Medical Care and Associate Dean Clinical Research, University of Southampton. He is also a general practitioner St Mary's Surgery, Southampton City Primary Care Trust. Robert Peveler is Professor of Liaison Psychiatry and Director of Clinical Neurosciences, University of Southampton, and Honorary Consultant and Non-Executive Director of Hampshire Partnership NHS Foundation Trust.

The National Institute for Health and Clinical Excellence (NICE) recently published an updated guideline on the management of depression in adults (CG90, www.nice.org.uk/CG90niceguideline) and a new guideline on the management of depression in adults with chronic physical health problems (CG91, www.nice.org.uk/ CG91niceguideline). Between them they update the guidance previously issued in $2004^{1}$ and introduce new recommendations in a number of areas. The two new guidelines are integrated in a single quick reference guide (http://guidance.nice.org.uk/ CG90/QuickRefGuide/pdf/English), which is the version sent out routinely to National Health Service (NHS) general practitioners (GPs). A quick reference guide to the identification and initial management of depression in patients with chronic physical health problems is also available for use in general hospital settings (www.nice.org.uk/CG91quickrefguide).

Key priorities for implementation are listed in the Appendix, showing a good deal of overlap between the two guidelines.

\section{Common issues}

Identification is displayed as a two-stage process, asking two validated questions about depression or lack of interest and pleasure in the past month, followed up by further questions only if patients answer 'yes' to one of them. The updated guidance continues to recommend a stepped care approach (reduced from five to four steps), but 'active monitoring' replaces 'watchful waiting' as recommended in the 2004 guidance, to emphasise that all patients with symptoms should be actively reviewed, normally within 2 weeks.

The new suggestion to consider using a validated symptom questionnaire when assessing severity rationalises the guidance with the GP contract quality and outcomes framework, which since 2006 has rewarded the use of questionnaires at diagnosis. ${ }^{2}$ In support of the recommendation, the CG90 guideline group carried out an extensive review of commonly used measures. However, the basis for assessment of severity has been switched in the updated guidance from the ICD $-10^{3}$ classification to the DSM-IV-TR. ${ }^{4}$ Therefore the threshold is now five rather than four symptoms, and the requirement that these are accompanied by functional impairment, implicit in DSM-IV, ${ }^{5}$ is made explicit in the updated guidance, along with a minimum duration. The 2004 guidance was criticised for a reliance on using symptom counts to make a diagnosis, ${ }^{6}$ and the greater emphasis on functional impairment and duration is helpful, given concerns that inexperienced GPs might rely only on a symptom score. ${ }^{7,8}$

A significant change is that drug treatment is now recommended for subthreshold depression which has lasted more than 2 years (calling this 'persistent symptoms' rather than dysthymia), which means the guidance is now consistent with the British Association for Psychopharmacology guidelines. ${ }^{9}$

The quick reference guide emphasises the need to take into account the views of patients and carers, particularly in terms of treatment choices, more strongly than the 2004 guideline. There is specific advice on building relationships, providing information, reducing jargon, advising about side-effects, offering referral to self-help groups, and obtaining advanced decisions about the acceptability of treatments in severe cases. There is much more in the full depression guideline CG90 on patients' and carers' experiences of depression than there was in the 2004 guideline (40 pages instead of 4), and a number of recent qualitative studies are reviewed there.

However, despite this increased emphasis on the individual experience of depression, the guidelines still adopt an essentially medical model, albeit with psychological therapies as one type of intervention. This is inherent in the underlying NICE framework, which starts from a medical model and relies for its evidence primarily on the results of randomised controlled trials. In common with all other national guidelines on depression, they do not address to any significant extent social issues which may affect the likely response to treatment in individual patients such as adverse life events or social support. ${ }^{10} \mathrm{~A}$ number of the recommendations do touch on social aspects that might be taken into account. However, there is a need for significantly more research into differences between patients, to inform the tailoring of recommendations based on evidence from groups of standardised patients (in the limited context of trials) to individual patients treated in a wider range of contexts in practice, which of course is a challenge to all of us working in this field. ${ }^{11}$

The 2004 guidelines were criticised for being unlikely to lead to improved outcomes for depression in the absence of considerable 
increases in organisational support for patients such as case management and collaborative care. ${ }^{12}$ The 2009 guideline groups have updated and reviewed the evidence on case management, medication management and collaboration with secondary care professionals in considerable detail. Although the updated general guideline CG90 refrains from making any recommendations on providing case management and collaborative care routinely for all adults with depression (given the mostly small effect sizes in the trials reported so far), CG91 now recommends collaborative care for more severe depression in people who also have chronic physical health problems (where the evidence was considered more convincing), while acknowledging that very little of the evidence is derived from a UK context. Unfortunately, it gives little detail on how this might be implemented for people whose care is largely being delivered by specialist services, and it is hard to see how this can easily be done given current NHS structures, with primary and secondary care services being deeply separated by formal contractual arrangements which make joint work and collaboration challenging. This challenge needs to be urgently addressed by primary care and mental health trusts, with support from their strategic health authorities.

Individual cognitive-behavioural therapy (CBT) is recommended as the first choice high-intensity psychological intervention for depression in the general adult guideline, as it was in the 2004 version, but group CBT, interpersonal therapy, behavioural activation, behavioural couples therapy, and mindfulness-based cognitive therapy are also recommended in the updated guidance where appropriate. The CG90 guideline group's review of the less convincing evidence for counselling and short-term psychodynamic psychotherapy leads to recommendations that these therapies have only a very limited role. In response, the patient group MIND has expressed fears that counselling may be squeezed out of NHS provision, reducing patient choice.

However, the wider range of evidence-based therapies reviewed and recommended gives commissioners of psychological therapies greater scope and could considerably enhance choice. One aspect of the success of the 2004 guidelines was in influencing the government to provide more CBT through the Increasing Access to Psychological Therapies (IAPT) programme, and the broadened recommendations on psychological therapies in the updated guidance could hopefully support further expansion of the IAPT programme at a time when financial pressures are mitigating against this. For example, training for the provision of more interpersonal therapy is being rolled out through the IAPT programme, but much work remains to be done on this.

One significant shortcoming of the NICE guidelines is that advice on common mental disorders is divided between a number of separate guidelines on depression and anxiety, yet people often present with comorbid depression, anxiety and physical illnesses in primary care. Having a number of different guidelines is not user-friendly to busy GPs trying to decide how to assess and treat someone with a common mental disorder. However, NICE have recognised the need for a more integrated guideline on case identification, assessment and care pathways for people with common mental disorders, and a new guideline development group on these aspects has recently been set up by the National Collaborating Centre for Mental Health.

\section{Issues for managing depression in people with physical illness}

The main issue for CG91 is that the evidence base in physical illness is much less well-developed, requiring 'extrapolation' from the broader evidence base in CG90, but this approach is made transparent (Table 4, p. 98). The scope of physical illnesses reviewed lists cancer, heart disease, musculoskeletal disorders, diabetes, respiratory and neurological disorders. There is explicit acknowledgement of the overlap that exists with guidelines such as those for diabetes and for renal disease, which also discuss management of depressive symptoms.

There is useful up-to-date information on the evidence of therapeutic benefit and harms of antidepressants in people with physical illness, who are often receiving additional treatments. Citalopram or sertraline are suggested as the first choice of antidepressant, with a helpful table on common drug interactions, and specific advice on reducing the risk of side-effects such as gastrointestinal bleeding and withdrawal symptoms. There is also considerably increased advice on sequencing treatments after an inadequate response, which is helpful, although the guidelines are not as comprehensive a source as specialist publications such as the British National Formulary or Maudsley Prescribing Guidelines.

Guideline CG91 also highlights the relative paucity of evidence concerning the impact of depression treatment on outcomes of the physical disease, for example whether treating depression benefits outcomes such as glycaemic control in diabetes, and whether there are differential effects of medication $v$. psychological treatments. As with all NICE guidance, focusing the research agenda is an important benefit of such a comprehensive review.

\section{NICE work}

So will the 2009 guidelines improve the care of depression? Hopefully yes, as they should encourage GPs to identify more people who will benefit from treatment, help optimise the use of antidepressants, and promote the further development and dissemination of psychological treatments, as did the 2004 guideline. However, they leave unaddressed the question of how they can best be implemented in current NHS structures, and the real test of the guidance will be the extent to which providers of acute and longer-term care for people with depression, with or without physical illness, respond to the recommendations.

\footnotetext{
Tony Kendrick, BSC, MD, FRCGP, FRCPsych, Primary Medical Care, Community Clinical Sciences Division, University of Southampton, and St Mary's Surgery, Southampton City Primary Care Trust; Robert Peveler, MA, DPhil, FRCPsych, Mental Health Group, Clinical Neurosciences Division, University of Southampton, and Hampshire Partnership NHS Foundation Trust, UK

Correspondence: Professor Tony Kendrick, Primary Medical Care, University of Southampton, Aldermoor Health Centre, Aldermoor Close, Southampton S016 5ST, UK. Email: ark1@soton.ac.uk

First received 10 Mar 2010, final revision 11 Mar 2010, accepted 23 Mar 2010
}

\section{Appendix}

Key priorities for implementation of the new NICE depression guidelines CG90 and CG91.

- Asking two questions about depression or a lack of interest and pleasure during the past month should be considered for people with a past history of depression, or with chronic physical health problems associated with functional impairment.

- A comprehensive assessment of patients with possible depression should be carried out which does not rely on a simple symptom count, but takes into account the degree of functional impairment and/or disability. 
- People with subthreshold symptoms, or mild to moderate depression, should be offered low-intensity psychosocial interventions, including guided self-help based on the principles of cognitive-behavioural therapy (CBT), computerised CBT, and/or a structured group physical activity programme.

- Antidepressant drug treatment should be considered for patients with subthreshold or mild depression where there is a past history of moderate or severe depression, and where subthreshold symptoms or mild depression have persisted for more than 2 years, or not responded to low-intensity psychosocial interventions.

- Psychological and psychosocial interventions should be based on manuals, and delivered by competent practitioners, with regular high-quality supervision, evaluating adherence by taping/external scrutiny, and monitoring outcomes with routine measures.

\section{Guidance for depression in adults (CG90) priority recommendations}

- Patients should be advised that antidepressants are not addictive and encouraged to continue medication for at least 6 months after remission.

- Cognitive-behavioural therapy should be offered to those who relapse or fail to recover with drug treatment alone.

- A combination of antidepressants and manualised psychologica therapy (CBT or interpersonal therapy) should be offered to patients with moderate to severe depression.

- Mindfulness-based cognitive therapy to prevent relapse should be considered where there have been three or more previous episodes of depression.

\section{Guidance for adults with a chronic physical health problem (CG91) priority recommendations}

- Antidepressants should be considered for mild depression that complicates the care of the physical health problem.

- When an antidepressant is prescribed, the presence of physical health disorders should be taken into account, including antidepressant sideeffects which may affect the underlying physical disease (in particular hyponatraemia from selective serotonin reuptake inhibitors in older people), and interactions with other medications.
- Group-based CBT or behavioural couples therapy (if relevant) should be considered for patients with moderate depression.

- Collaborative care should be considered for moderate to severe depression if the depression has not responded to CBT or interpersonal therapy, antidepressant drug treatment, or a combination of both psychological and drug treatment.

\section{References}

1 National Collaborating Centre for Mental Health. Depression: Management of Depression in Primary and Secondary Care. Clinical Guideline 23. National Institute for Health and Clinical Excellence, 2004.

2 British Medical Association, NHS Employers. Revisions to the GMS Contract 2006/07. Delivering Investment in General Practice. Department of Health, 2006.

3 World Health Organization. International Statistical Classification of Diseases and Related Health Problems, 10th Revision (ICD-10). WHO, 1997.

4 American Psychiatric Association. Diagnostic and Statistical Manual of Mental Disorders, (4th edn, text revision) (DSM-IV-TR). APA, 2000.

5 American Psychiatric Association. Diagnostic and Statistical Manual of Mental Disorders (4th edn) (DSM-IV). APA, 1994

6 Middleton $\mathrm{H}$, Shaw I, Hull S, Feder G. NICE guidelines for the management of depression. BMJ 2005; 330: 267-8.

7 Dowrick C, Leydon GM, McBride A, Howe A, Burgess H, Clarke P, et al. Patients' and doctors' views on depression severity questionnaires incentivised in UK quality and outcomes framework: qualitative study. BMJ 2009; 338: b663.

8 Kendrick T, Dowrick C, McBride A, Howe A, Clarke P, Maisey S, et al. Management of depression in UK general practice in relation to scores on depression severity questionnaires: analysis of medical record data. BMJ 2009; 338: b750.

9 Anderson IM, Ferrier IN, Baldwin RC, Cowen PJ, Howard L, Lewis G, et al. Evidence-based guidelines for treating depressive disorders with antidepressants: a revision of the 2000 British Association for Psychopharmacology guidelines. Psychopharmacology 2008; 22: 343-96.

10 Hegarty K, Gunn J, Blashki G, Griffiths F, Dowell T, Kendrick T. How could depression guidelines be made more relevant and applicable to primary care? Br J Gen Pract 2009; 59: e149-56.

11 Kendrick T, Hegarty $K$, Glasziou P. Interpreting research findings to guide treatment in practice. BMJ 2009; 337: a1499.

12 Whitty P, Gilbody S. NICE, but will they help people with depression? The new National Institute for Clinical Excellence depression guidelines. $\mathrm{Br} J$ Psychiatry 2005; 186: 177-8. 